\title{
Tabularia
}

TABULARIA Sources écrites des mondes normands médiévaux

\section{Le Traité de Gaillon (1196) : Édition critique et traduction}

The Treaty of Gaillon (1196): critical edition and translation

\section{Emmanuel Rousseau et Gilles Désiré dit Gosset}

\section{OpenEdition}

\section{Journals}

Édition électronique

URL : http://journals.openedition.org/tabularia/2461

DOI : 10.4000/tabularia.2461

ISSN : $1630-7364$

Éditeur :

CRAHAM - Centre Michel de Boüard, Presses universitaires de Caen

\section{Référence électronique}

Emmanuel Rousseau et Gilles Désiré dit Gosset, « Le Traité de Gaillon (1196) : Édition critique et traduction », Tabularia [En ligne], Sources en ligne, mis en ligne le 05 novembre 2002, consulté le 21 avril 2019. URL : http://journals.openedition.org/tabularia/2461; DOI : 10.4000/tabularia.2461 


\title{
Le Traité de Gaillon (1196) : édition critique et traduction
}

\section{The Treaty of Gaillon (1196): critical edition and translation}

\author{
Emmanuel ROUSSEAU \\ et Gilles DÉSIRÉ dit GOSSET \\ Archives départementales de la Manche, \\ 103, rue du Maréchal Juin, \\ BP 54050010 Saint-Lô cedex \\ gilles.desire@cg50.fr \\ Mots-clés : paix, Gaillon, Philippe-Auguste, Richard Cour-de-Lion. \\ Keywords: peace, Gaillon, Philip Augustus, Richard Lionheart.
}

Dans le conflit qui oppose Richard Cour-de-Lion et Philippe-Auguste, le traité de Gaillon marque une courte période de trêve avant la reprise de la guerre presqu'ininterrompue qui oppose les deux souverains entre le retour d'Allemagne de Richard (mars 1194) et sa mort devant Châlus (avril 1199). La guerre a pour théâtre la Normandie et le Berry; alors que les deux rois s'apprêtent à en découdre sous les murs d'Issoudun, les pressions du clergé, alarmé par une menace d'invasion de l'Espagne par les musulmans, les obligent à conclure une trêve, bientôt transformée en paix.

Le traité est conclu entre Gaillon et Le Vaudreuil. Bien qu'il ne soit pas explicitement daté, on retiendra celle du 14 janvier 1196, puisque les conventions prises entre les deux souverains, lors de leur rencontre le 5 décembre, entre Chârost et Issoudun, donnent la Saint Hilaire comme terme de la signature ${ }^{1}$.

C'est une paix de compromis : Philippe conserve une partie de ses conquêtes normandes (le Vexin normand, Gisors, Vernon, Nonancourt, Pacy notamment) et voit reconnue l'affirmation de sa suzeraineté sur l'Auvergne; en revanche, il doit abandonner à Richard le Berry aquitain et bon nombre de places fortes comme Issoudun et Graçay. Cette entente ne satisfait réellement aucune des deux parties et la guerre reprend dès l'été 1196.

1. Daniel, Gabriel, Histoire de France depuis l'établissement de la monarchie françoise dans les Gaules..., Nouvelle édition..., t. 4, Paris, 1755, p. 98. 
Le traité de Gaillon nous est parvenu sous la forme de deux actes échangés entre les deux souverains, l'un au nom de Richard, l'autre au nom de Philippe-Auguste. C'est dans le Trésor des chartes aux Archives nationales (J 628, n $14-$ AE III, 62) qu'est conservé le premier, celui qui fait l'objet de la présente édition. Cet acte original sur parchemin (290 x 270 mm.), scellé du sceau de cire brune de Richard ${ }^{2}$, pendant sur lacs de soie rouge, est remarquablement conservé. Les historiens l'ont repéré depuis longtemps ${ }^{3}$ et il a déjà fait l'objet de nombreuses éditions ainsi qu'en témoigne la liste suivante:

Du CHESNE, André, Scriptores antiqui, res ab illis per Galliam, Angliam, Apuliam, Capuae principatum, Siciliam \& Orientem gesta explicantes, ab anno Christi DCCCXXXVIII ad annum MCCXX, Paris, 1619, p. 1053-1055.

DumONT, Jean, Corps universel diplomatique du droit des gens..., Amsterdam, La Haye, 1726, t. 1, pars 1, p. 119, $n^{\circ}$ CCXV.

BRUSSEL, Nicolas, De l'usage des fiefs, t. II, Paris, 1750, p. XIII-XVII.

BRIAL, Michel-Jean-Joseph (Dom), Recueil des historiens des Gaules et de la France, t. XVII, Paris, 1818, p. 43-45.

DELISLE, Léopold, "Cartulaire normand de Philippe-Auguste, Louis VIII, saint Louis et Philippe-le-Hardi», Mémoires de la Société des Antiquaires de Normandie, $2^{e}$ série, t. 16, $n^{\circ}$ 1057, Caen, A. Hardel, 1852, p. 276-277.

TeUlet, Alexandre, Layettes du Trésor des Chartes, t. I, Paris, Plon, 1863, $n^{\circ} 431$, p. 182-184.

BONnIN, Théodose, Cartulaire de Louviers, documents historiques originaux $d u X^{e}$ au XVIII ${ }^{e}$ s..., t. I, Evreux, Leclerc, 1870, $n^{\circ}$ LV, p. 71-74.

FAGNEN, Claude, Essai sur quelques actes normands de Richard Cour-de-Lion, thèse de l'Ecole des chartes, 1971, t. 3, p. 469-478, nº 98.

Gabriel du Moulin, dans son Histoire générale de Normandie ${ }^{4}$ en a esquissé une traduction dès la première moitié du XVII ${ }^{\mathrm{e}}$ siècle.

De même, l'acte au nom de Philippe-Auguste a connu un succès presqu'égal chez les diplomatistes des XVIII ${ }^{\mathrm{e}}$ et XIX ${ }^{\mathrm{e}}$ siècles :

- RYMER, Thomas, Foedera, conventiones, literae et cujuscumque generis acta publica inter reges Anglie et alios quosvis..., $3^{e} e ́ d .$, t. I, part. 1, Hagae Comitis,

2. DouËT D'ARCQ, Louis, Collection de sceaux, Paris, Plomb, 1863-1868, 3 vol.; n 10007-10 007 bis. Sceau rond de $100 \mathrm{~mm}$ de diamètre; type de majesté, avec l'épée à la main droite, et à la gauche le globe, qui est surmonté d'une tige terminée par une croix. De chaque côté de la tête, un soleil et un croissant; de chaque côté du trône, une branche de trois fleurs. Légende: (en onciale) RICARDUS DEI GRACIA REX ANGLORUM. Le contre-sceau est un type équestre; sur le bouclier, un lion rampant. Légende: (en onciale) RICHARDUS DUX NORMANNORUM ET AQUITANORUM ET COMES ANDEGAVORUM.

3. Signalé dans Delisle, Léopold, Catalogue des actes de Philippe-Auguste, Paris, 1856, p. 110, $\mathrm{n}^{\circ} 464$.

4. Du Moulin, Gabriel, Histoire générale de la Normandie..., Rouen, 1631, p. 480-482. 
1745, 29-30.

- RYMER, Thomas, Foedera, conventiones, litterae... éd. de la Record Commission, 1816, t. I, part. 1, p. 66.

- BOUQUET, Martin (Dom), Recueil des Historiens des Gaules et de la France, t. XVIII, Paris, 1822, p. 50-525.

- BonNIN, Théodose, Cartulaire de Louviers, documents historiques originaux du Xe au XVIII ${ }^{e}$ s..., t. I, Evreux, Leclerc, 1870, $n^{\circ}$ LVI, p. 74-77.

- Recueil des actes de Philippe-Auguste, roi de France, t. II, Paris, Impr. Nationale, $1943, n^{\circ} 517$, p. 52-576.

Cependant, l'analyse de ces différentes éditions, déjà anciennes, montrent qu'elles sont souvent fautives. De plus, ce très long traité fourmille de noms de lieux et de personnages parfois difficiles à identifier. C'est pourquoi, à la demande du musée de l'histoire de France et dans le cadre de travaux pratiques à l'Ecole nationale des chartes, nous avons été amenés à éditer à nouveau cet acte et à le traduire, en identifiant les noms propres de la façon la plus précise possible.

\section{Édition}

Ricardus, Dei gratia, rex Anglie, dux Normannie et Aquitanie, comes Andegavie, omnibus ad quos littere presentes pervenerint salutem in Domino. Nosse vos volumus quod hee sunt conventiones pacis inter nos et dominum nostrum Philippum, illustrem $/ 2$ / regem Francorum ${ }^{7}$, facte in vigilia sancti Nicolai, inter Exoldunum et Charrocium, videlicet: quod eidem et heredibus suis, jure hereditario in perpetuum dimittimus et quitamus Gisorcium et Nielpham et Velcassinum Normannum; ita quod Stephanus de Longo-Campo debet ha-/3/-bere Baldemont et terram suam et tenebit eam de rege Francorum. De Hugone de Gornai ita erit: hommagium ejus remanet regi Francorum ad vitam dicti Hugonis, nisi voluerit redire ad nos. Et post mortem ejusdem Hugonis debet /4/ totum feodum suum de Normannia ad nos et heredes nostros redire. Terra ejusdem Hugonis quam habuit in Anglia et in Normannia, debet dari Ricardo de Vernone, pro excambio illo quod rex Francorum debet facere eidem Ricardo pro castello Vernonis, scilicet de oc-/5/-tingentis libris parisiensium de redditibus; ita quod si prefata terra Hugonis tantum non valeret per annum, rex Francorum ei in terra sua perficeret residuum. Ricardus autem et filius suus regi Francorum et heredibus suis Vernonem cum castellaria sua in /6/ perpetuum quitaverunt de mandato et assensu nostro et quitationem juraverunt. Preterea quitationem illam quam comes Lecestrie domino nostro Philippo, regi Francorum, fecit de castello Paciaci, tam in feodo quam in dominio, cum castella-

5. Édition d'après RYMER, $2^{\mathrm{e}}$ éd.

6. Mentionne les variantes par rapport à l'acte au nom de Richard Cœur-de-Lion.

7. Contrairement aux précédents éditeurs, nous suivons ici l'avis du Manuel de Diplomatique d'Arthur Giry (Paris, 1894, p. 320) qui indique que la titulature du roi de France a toujours été rex Francorum. 
ria sua /7/ et pertinentiis ipsius, ratam habemus et firmam. Preterea quitamus regi Francorum et heredibus suis in perpetuum, jure hereditario, Novum-Mercatum, Vernonem, Gallionem, Paciacum, Ivriacum, Nonencortem cum castellariis eorum. Et sciendum quod mete ponen-/8/-tur inter forteliciam Gallionis et forteliciam VallisRodolii in media via. Et ex illa meta, sicut se portabit, usque in Secanam, et ex alia parte usque in Euriam, id quod erit ex parte Gallionis erit regis Francorum et id quod erit ex par-/9/-te Vallis-Rodolii erit nostrum. Quitamus etiam eidem regi Philippo et heredibus suis in perpetuum, Alverniam, feodum et dominium, et quod habebamus ibi et quod nos habituros expectabamus. Preterea si comes Lecestrie aut Ricardus de Vernone, aut filius suus, aut /10/ aliquis ex hominibus nostris, occasione feodi et dominii que nos per hanc pacem eidem regi dimittimus, ipsi aut suis aliquod malum faceret, nos mitteremus in manus ejusdem regis Philippi terras eorum, et salvo eum tenere faceremus, usque dum dampna ipsi et suis restituta essent /11/ vel nos de nostro eidem regi et suis restitueremus, et illos de terra nostra fugaremus. Ut autem firma pax sit inter nos et dominum nostrum Philippum, regem Francorum, dimittit et quitat nobis et heredibus nostris in perpetuum idem rex Francorum Exoldunum et Graciacum, /12/ et omnia feoda que pertinent ad Exoldunum et Graciacum, et feodum de La Chastre et de Sancto Chartier et de Castelleto, sicut Andreas de Calviniaco tenebat ea de rege Francorum, et feodum Castelli-Meillant. [, sicut Odo de Dolis eum de rege Fran-/13/-cie tenebat; et Sellacum cum pertinentiis, eo excepto de quo comes Sancti Egidii et sui, aut vicecomes Turanie et sui in vigilia beati Nicolai erant tenentes. Si rex Francorum vellet firmare in Villanova super Cherum, firmare poterit. /14/ Nobis autem remanet comitatus Augi, cum omnibus pertinentiis suis, sicut comes Augi et sui homines habuerunt; comitatus de Albamarla cum pertinentiis suis; Arches et Driencort cum pertinentiis suis. Terre vero militum de terra Hugo-/15/-nis de Gornai qui venerunt ad nos reddentur illis, ita quod de terris illis facient hommagium et servicium Hugoni de Gornai, salva fidelitate quam ipsi nobis debebant. Dimittit etiam nobis rex Francorum Belveeir et officium de Belveeir, et totam /16/ terram aliam nostram et hominum nostrorum, quam amisimus postquam capti fuimus in Alemannia, exceptis predictis terris que remanent regi Francorum et heredibus suis in perpetuum, sicut dictum est. In uno et eodem puncto erimus nos et comes Sancti Egidii, quod /17/ nos tenebimus totam terram quam tenebamus in vigilia sancti Nicolai; et faciemus et inforciabimus in terra nostra quam tunc tenebamus, sicut voluerimus, tamquam de nostra: et comes Sancti Egidii similiter de sua quam tunc tenebat. Et si comes Sancti Egidii /18/ nollet esse in hac pace, dominus noster rex Francorum non erit ei in auxilium contra nos; et nos omnia mala que possemus ei facere, faceremus, et de incendio et de eversione. Et si nos vellemus tenere firmum id quod cepissemus, quando comes Sancti Egidii vellet /19/ esse in pace, nos redderemus ei totam terram suam quam a vigilia beati Nicolai acquisivissemus; et similiter erit de comite Sancti Egidii. Et si comes Sancti Egidii voluerit esse in hac pace, nos neque malum, neque guerram faciemus eidem comiti, quamdi-/20/-u idem comes voluerit rectum facere per regem Francorum. Comes Petragore habebit terram suam sicut habebat quando recessit a nobis; et similiter 
vicecomes Brocie, comes Engolismi, rehabebit terram suam, et homines sui et ipsi tres facient nobis homi-/21/-nagium et servitium sicut prius. Vicecomes Turanie tenebit de rege Francorum id quod debet et de nobis id quod debet. De Fortunato de Gorduno sic erit : quod si poterimus probare per sacramenta viginti vel triginta legitimorum hominum quod duo cas-/22/-tella, scilicet Caseaus et Perillac, tenuissemus per unum annum et unum diem, et amplius, et nos ea predicto Fortunato tradidissemus, si nos ea habere voluerimus, dominus noster rex Francorum inde se non intromittet. De domo Castelli-Novi Turonis edificanda, faciemus /23/ ad consilium archiepiscopi Remensis et Drogonis de Melloto. De Andeliaco sic erit: quod nec dominus noster rex Francorum nec nos in eo clamamus feodum sive dominium. Et si contigerit quod archiepiscopus Rothomagensis in terram regis Francorum aut suorum senten-/24/-tiam interdicti vel excommunicationis miserit, dominus rex Francorum poterit assignare ad Andeliacum et ad ea que archiepiscopus ibi habet, et ad ejus pertinentias, usque dum duo diaconi vel presbiteri, quos rex Francorum per sacramentum suum bona fide ad hoc elegerit, et duo dia-/25/coni vel presbiteri, quos nos per sacramentum nostrum bona fide ad hoc elegerimus, decreverint utrum interdictum vel excommunicatio juste latum fuerit an injuste. Si decreverint quod juste, rex Francorum predicto archiepiscopo reddet Andeliacum et ea que interim exinde /26/ levaverit, et ad verbum dictatorum faciet emendari. Si vero decreverint quod injuste positum fuerit, ea, que rex Francorum de Andeliaco et de pertinentiis ejus levaverit, in deperdito erunt archiepiscopi, et archiepiscopus interdictum vel excommunicationem solvet. Similiter erit de nobis. /27/ Si aliquis predictorum dictatorum moreretur, hinc vel inde, per sacramentum alterius nostrum alter loco mortui similiter supponetur. Quando archiepiscopum mori contigerit, redditus de Andeliaco et de pertinentiis erunt in manu capituli beate Marie /28/ Rothomagensis donec alius succedat archiepiscopus. Nec nos aliquod malum faciemus predictis dictatoribus, propter arbitrium ipsorum. Andeliacum non poterit inforciari. Nos domino nostro regi Francorum faciemus quitari omnia illa que cepit de rebus ecclesiarum terre nostre, que sunt in terra /29/ sua; et idem rex Francorum similiter nobis. Neque nos, neque rex Francorum de cetero, propter aliquam guerram que evenire possit, aliquid capiemus vel supercapiemus de rebus ecclesiarum, alter de terra alterius. Et in ea pace et libertate erunt ecclesie, hinc inde, in qua erant ante /30/ guerram. Amodo non intromittemus nos de hominibus regis Francorum, neque de feodis qui ad eum pertinent. Nec ipse de nostris; salvis tamen regis Francorum serviciis que ipsi debemus, de feodis quos ab ipso tenemus, sicut feudi apportant. Neque nos recipiemus /31/ amodo homines ligios regis Francorum contra ipsum quamdiu vixerit, nec ipse nostros homines ligios contra nos quamdiu vixerimus. Hiis omnibus peractis, comes Lecestrie, et omnes prisones, et ostagii prisonum, prout divisum est, hinc inde liberabuntur. Nos juravimus /32/ bona fide quod nos hec tenebimus, et rex Francorum similiter. Que omnia supradicta ut firma et stabilia permaneant, sigillo nostro confirmamus. Actum inter Gallionem et VallemRodolii, anno Incarnati Verbi millesimo centesimo nonagesimo quinto. 


\section{Traduction}

Richard, par la grâce de Dieu, roi d'Angleterre, duc de Normandie, d'Aquitaine, comte d'Anjou, à tous ceux auxquels les présentes lettres parviendront, salut dans le seigneur.

Nous voulons vous faire connaître que telles sont les conventions de paix passées entre nous et notre seigneur Philippe, illustre roi de France, la veille de la Saint Nicolas $^{8}$, entre Issoudun ${ }^{9}$ et Chârost ${ }^{10}$, à savoir que nous lui remettons et lui abandonnons, à lui et à ses héritiers, à perpétuité et en droit héréditaire, Gisors ${ }^{11}$, Neaufles ${ }^{12}$ et le Vexin normand ${ }^{13}$, de sorte qu'Etienne de Longchamps ${ }^{14}$ ait Baudemont ${ }^{15}$ et sa terre, et il la tiendra du roi de France.

Au sujet de Hugues de Gournay ${ }^{16}$, il en sera ainsi : son hommage reste au roi de France pendant toute la vie dudit Hugues, à moins qu'il ne veuille revenir à nous. Et, après la mort dudit Hugues, tout son fief en Normandie doit nous revenir, à nous et à nos héritiers.

La terre que ledit Hugues avait en Angleterre et en Normandie, doit être donnée à Richard de Vernon ${ }^{17}$, en vertu de l'échange que le roi de France doit faire avec

8. Cette fête étant fixée au 6 décembre, les conventions du traité peuvent donc être datées du 5 .

9. Exoldunum. Issoudun, Indre, ch.-1. arr.

10. Carrocium. Chârost, Cher, arr. Bourges, ch.-1. cant. Du Moulin, Histoire générale de la Normandie, p. 480, indique à tort Châteauroux dans sa traduction.

11. Gisorcium. Gisors, Eure, arr. Les Andelys, ch.-l. cant.

12. Nielpha. Neaufles-Saint-Martin, Eure, arr. Les Andelys, cant. Gisors.

13. Velcassinum Normannum. Vexin normand.

14. Stephanus de Longo Campo. Etienne de Longchamps (Eure, arr. Les Andelys, cant. Etrépagny), seigneur normand, issu d'une famille attestée au XI ${ }^{\mathrm{e}}$ s. et éteinte à la fin du XIII'. Il est plusieurs fois cité dans le Grand Rôle de l'Echiquier de Normandie de 1198 (éd. M.S.A.N., 2 série, t. 16, 1852, p. 49, 50b, 52, 67b), dans celui de 1203 (ibid., p. 100), dans Delisle, Cartulaire normand, en 1205 ( ${ }^{\circ} 124$ ) et en 1213 ( $\mathrm{n}^{\circ} 230$ et 1109). Voir son sceau dans Doüet d'Arcq, $\mathrm{n}^{\circ} 2608$. Après le traité, Richard, ayant repris une partie du Vexin normand, lui rendit le château de Longchamps, qu'il conserva jusqu'à sa reprise par Philippe-Auguste en 1202. Il assistait à l'Echiquier de 1214 et fut tué la même année à Bouvines. Bibliogr.: Charpillon, Dictionnaire historique de toutes les communes du département de l'Eure..., t. II, Les Andelys, 1879, p. 448-449.

15. Baldemont. Baudemont, Eure, arr. Les Andelys, cant. Ecos.

16. Hugo de Gornai. Hugues IV de Gournay (-en-Bray, Seine-Maritime, arr. Dieppe, ch.-l. cant.), fils de Hugues III $(\dagger 1180)$ et de Mélissandre de Coucy, époux de Julienne de Dammartin, ami proche de Richard Coeur-de-Lion, appartenait à une très ancienne maison remontant à Eudes, qui reçut son fief de Gournay des mains de Rollon. Dans la suite du conflit franco-anglais, il prit parti pour Jean-sansTerre et, après confiscation de ses biens, ne dut son salut qu'à la fuite en Angleterre, où ses descendants subsistaient au XIX ${ }^{\mathrm{e}}$ siècle. Bibliogr.: BUNEL, Joseph, continué par TOUGARD, Albert, Géographie du département de la Seine Inférieure, arr. de Neufchatel, Rouen, Cagniard, 1875, p. 156-159; GURNEY, Daniel, The record of the House of Gournay, compiled from original documents, 2 vol., Londres, Nichols, 1848-1858.

17. Ricardus de Vernone. Richard II de Vernon appartient à l'illustre maison de Vernon et Reviers, issue de Richard ${ }^{\text {er }}$, duc de Normandie. Il était fils de Guillaume II de Vernon et de Reviers († 1160) et de 
ledit Richard pour le château de Vernon ${ }^{18}$, à savoir huit cents livres parisis de rente; de sorte que si la terre dudit Hugues ne rapportait pas autant chaque année, le roi de France verserait le complément sur sa propre terre. Richard et son fils ont renoncé à perpétuité en faveur du roi de France et de ses héritiers à Vernon et sa châtellenie, sur notre ordre et accord, et ils ont juré cette renonciation ${ }^{19}$.

En outre, nous confirmons et approuvons la renonciation faite par le comte de Leicester ${ }^{20}$ en faveur de notre seigneur Philippe, roi de France, du château de Pacy ${ }^{21}$, tant fief que seigneurie, avec sa châtellenie et ses dépendances ${ }^{22}$.

En outre, nous abandonnons au roi de France et à ses héritiers, à perpétuité, en droit héréditaire, Neuf-Marché ${ }^{23}$, Vernon, Gaillon ${ }^{24}$, Pacy, Ivry ${ }^{25}$, Nonencourt ${ }^{26}$ avec leurs châtellenies. Et qu'on sache qu'on mettra des bornes à mi-chemin, entre la forteresse de Gaillon et celle du Vaudreuil ${ }^{27}$; et de la première borne, où elle se trouvera, jusqu'à la Seine ${ }^{28}$, et d'autre part jusqu'à l'Eure ${ }^{29}$, tout ce qu'il y aura du côté de Gaillon sera au roi de France, et tout ce qu'il y aura du côté du Vaudreuil sera à nous.

Nous abandonnons également audit roi Philippe et à ses héritiers, pour toujours, l'Auvergne ${ }^{30}$, fief et seigneurie, ce que nous y avons et ce que nous espérions y avoir.

En outre, si le comte de Leicester ou Richard de Vernon, ou son fils, ou l'un de nos hommes, sous prétexte de leur fief et seigneurie que nous abandonnons audit roi par cette paix, causaient du tort au roi ou aux siens, nous remettrions leurs terres entre les mains du roi Philippe, et nous veillerions à ce qu'il les tienne en sureté, jusqu'à ce que les torts causés à lui et aux siens fussent réparés, ou bien nous donnerions audit roi et aux siens une partie de nos terres, et nous chasserions les susdits de nos terres.

Pour qu'il y ait une paix stable entre nous et notre seigneur Philippe, roi de France, ledit roi de France nous donne et nous abandonne à nous et nos héritiers,

Lucie de Tancarville, père de Richard III de Vernon, connétable de Normandie ( $†$ ap. 1226) avec qui s'éteint la branche française de cette famille. Bibliogr.: CHARPILlon, Dictionnaire..., p. 970972; MEYER, Edmond, Hist. de la ville de Vernon, 2 vol., Les Andelys, Delcroix, 1874-76.

18. Verno. Vernon, Eure, arr. Evreux, ch.-l. cant.

19. Les actes d'échange et de renonciation dudit Richard sont cités par Léopold Delisle dans son Cartulaire normand $\left(\mathrm{n}^{\circ} 33-34\right)$.

20. Robert, dit "fils de Perronnelle", comte de Leicester (Co-Hst, Angleterre) de 1190 à sa mort en 1204.

21. Paciacum. Pacy-sur-Eure, Eure, arr. Evreux, ch.-1. cant.

22. Cette renonciation fit l'objet de plusieurs actes mentionnés par Léopold Delisle dans son Cartulaire normand ( $\mathrm{n}^{\circ} 36$ à 41$)$.

23. Novum-Mercatum. Neuf-Marché, Seine-Maritime, arr. Dieppe, cant. Gournay-en-Bray.

24. Gallionis. Gaillon, Eure, arr. Les Andelys, ch.-l. cant.

25. Ivriacum. Ivry-la-Bataille, Eure, arr. Evreux, cant. Saint-André.

26. Nonencortem. Nonancourt, Eure, arr. Evreux, ch.-l. cant.

27. Vallis-Rodolii. Le Vaudreuil, Eure, arr. Les Andelys, ch.-l. cant.

28. Secana. La Seine.

29. Euria. L'Eure.

30. Alvernia. L'Auvergne. 
pour toujours, Issoudun et Graçay ${ }^{31}$ et tous les fiefs qui dépendent d'Issoudun et Graçay; et les fiefs de la Châtre ${ }^{32}$, de Saint-Chartier ${ }^{33}$ et du Châtelet ${ }^{34}$, comme André de Chauvigny ${ }^{35}$ les tenait du roi de France; et le fief de Châteaumeillant ${ }^{36}$ comme Eudes de Déols ${ }^{37}$ le tenait du roi de France; et Souillac ${ }^{38}$ avec ses dépendances, sauf ce que le comte de Saint-Gilles ${ }^{39}$ et les siens, ou le vicomte de Turenne ${ }^{40}$ et les siens tenaient à la veille de la Saint Nicolas ${ }^{41}$.

31. Craciacum. Graçay, Cher, arr. Bourges, ch.-1. cant.

32. La Chastre. La Châtre, Indre, ch.-l. arr.

33. Sanctus Chartier. Saint-Chartier, Indre, arr. et cant. La Châtre.

34. Castelletum. Le Châtelet, Cher, arr. et cant. Saint-Amand-Montrond. Dom Brial, op. cit., p. 44, $\$ 8$, indique par erreur Châtillon-sur-Cher (Loir-et-Cher, arr. Blois, cant. Saint-Aignan).

35. Andreas de Calviniaco. André de Chauvigny (Vienne, arr. Montmorillon, ch.-l. cant.), surnommé Chauvigny le Clop (c'est-à-dire le boîteux), seigneur de Montoiron, fils de Pierre-Hélie de Chauvigny, dont la famille tenait la prévôté héréditaire de Chauvigny pour le compte des évêques de Poitiers, et de Haoïs de Chatellerault, la grand-tante de Richard Coeur-de-Lion. Il devint seigneur de Déols, Le Châtelet, La Châtre et Saint-Chartier en 1189 par son mariage avec Denise de Déols (1173-1207), fille et héritière de Raoul VII de Déols (†1176). Il porta son hommage à Philippe-Auguste en octobre 1199 (Arch. nat., J 399 n ${ }^{\circ}$ ) après la mort de Richard. Assassiné après sa capture à la bataille de Mirebeau (1202) où il s'était allié avec Arthur de Bretagne contre Jeansans-Terre, il est devenu le héros d'une chanson de geste. Il était par ailleurs seigneur du fief de Bituresii, dont Jean-sans-Terre abandonne la suzeraineté au roi de France en mai 1200 (Cartulaire normand, $\mathrm{n}^{\circ}$ 1063). La Chenaye-Desbois et Badier (Dictionnaire de la noblesse, t. 6, col. 819) le qualifient de "généralissime" des troupes de Philippe-Auguste en Terre sainte; c'est en effet l'un des héros de la troisième croisade. Bibliogr.: HUBERT, Eugène, Le Bas-Berry, histoire et archéologie du dép. de l'Indre, Chateauroux et Déols, fasc. 1, Paris, 1930, p. 71-94 ; MEUNIER, J.-J., "Les Maisons de Déols et de Chauvigny (952 - 1503) ", Revue de l'Académie du Centre, Châteauroux, 1965, p.30-60; Europäische Stammtafeln, Band XIII, Marburg, 1990, Tafel 70; DouËT D'ARCQ, $\mathrm{n}^{\circ} 1818$.

36. Castellum Meillant. Châteaumeillant, Cher, arr. Saint-Amand-Montrond, ch.-l. cant.

37. Odo de Dolis. Eudes de Déols († 1208), second fils d'Ebbe II (1141-1160), époux d'Agnès († 1220), reçut Châteaumeillant en 1160 puis le château de Boussac après la mort de son oncle Geoffroy de Preuilly. Le fief de Châteaumeillant était entré dans cette maison par le mariage du grand-père d'Eudes, Raoul VI de Déols (1099-1141), avec Beatrix “Fenion”, héritière d'Alard Guillebaud, "prince du château de Saint-Chartier" et seigneur de Châteaumeillant ( $†$ ap. 1122). Cette branche de la maison de Déols tombe en quenouille après la mort des fils d'Eudes, Raoul (ap. 1218) et Ebbe III (1256). Bibliogr.: Hubert, Eugène, Le Bas-Berry..., p. 57-67. Du Moulin, Gabriel, Histoire générale de Normandie, p. 481, § VIII, l'appelle Eudes de Dol.

38. Sellacum. Souillac, Lot, arr. Gourdon, ch.-l. cant. DU Moulin, Gabriel, Histoire générale de Normandie, p. 481, \$ VIII, indique "Sellat".

39. Raymond VI de Saint-Gilles, né le 27 octobre 1156, devint le $22^{\mathrm{e}}$ comte de Toulouse en 1194, à la mort de son père, Raymond V. Il épouse en quatrième noces Jeanne d'Angleterre († 1199), soeur de Richard Coeur-de-Lion (12 novembre 1196), qui lui donne le futur Raymond VII le 20 juillet 1197. Il meurt le 22 septembre 1222. Bibliogr. : GENTY, Roger, Les comtes de Toulouse : histoire et traditions, Ferrières, Ed. de Poliphile, 1987, p. 183-202.

40. Vicecomes Turanie. Raymond III ( $† 12$ juin 1219), vicomte de Turenne, fils de Raymond II ( $\dagger$ 1191 devant Acre) et de Hélie de Castelnau, époux de Hélie de Séverac. Bibliogr.: Europäische Stammtafeln, Band III (4), Tafel 766. Du Moulin, Gabriel, Histoire générale de Normandie, p. 481, $\$$ VIII, traduit, probablement par erreur de lecture: "le comte de Turame".

41. Du Moulin, Gabriel, Histoire générale de Normandie, p. 481, \$ VIII, indique par erreur: "en la S. Michel derniere". 
Si le roi de France voulait fortifier Villeneuve-sur-Cher ${ }^{42}$, il le pourrait.

Il nous reste le comté d'Eu ${ }^{43}$ avec toutes ses dépendances, comme le comte d'Eu ${ }^{44}$ et ses hommes l'ont reçu; le comté d'Aumale ${ }^{45}$ avec ses dépendances; Arques ${ }^{46}$ et Neufchâtel ${ }^{47}$ et leurs dépendances.

Mais les terres des chevaliers de la terre de Hugues de Gournay, qui sont venus auprès de nous, leur seront rendues, de sorte qu'ils rendront hommage et service pour ces terres à Hugues de Gournay, la fidélité qu'ils nous devaient restant sauve.

Le roi de France nous abandonne en plus Beauvoir ${ }^{48}$ et la circonscription de Beauvoir et toutes nos autres terres, et celles de nos hommes, que nous avons perdues après que nous avons été capturé en Allemagne, excepté les terres susdites qui restent au roi de France et à ses héritiers, pour toujours, comme il est dit.

Nous en resterons au même point avec le comte de Saint-Gilles, à savoir que nous garderons toutes les terres que nous tenions à la veille de la Saint Nicolas; et nous agirons et nous fortifierons sur cette terre que nous tenions jusqu'alors, comme nous le voudrons, comme si elle était nôtre: et le comte de Saint-Gilles agira de même avec la terre qu'il tenait jusqu'alors. Et si le comte de Saint-Gilles refusait de participer à cette paix, notre seigneur le roi de France refusera de lui venir en aide contre nous; et nous, nous lui ferions tout le tort que nous pourrions, tant par incendie que par subversion. Et si nous voulions tenir fermement ce que nous avons pris quand le comte de Saint-Gilles voulait être en paix, nous lui rendrions toute sa terre que nous aurions prise depuis la veille de la Saint Nicolas ${ }^{49}$; et le comte de Saint-

42. Villanova super Cherum. Villeneuve-sur-Cher, Cher, arr. Bourges, cant. Chârost.

43. Comitatus Augi. Comté d'Eu (Seine-Maritime, arr. Dieppe, ch.-1. cant.).

44. La maison des comtes d'Eu, issue de Richard I ${ }^{\text {er }}$, duc de Normandie, tombe en quenouille après la mort d'Henri II, comte d'Eu, en Terre Sainte (1192 ou 1194) et de ses deux fils, Raoul et Gui (1222). Le comté passe alors entre les mains de sa fille Alix (†1246) qui épouse Raoul de Lusignan (†1214 ou 1218), frère de Gui de Lusignan, roi de Jérusalem. Bibliogr. : EsTANCELIN, Louis, Histoire des comtes d'Eu, Rouen, impr. de Mogard père,1828.

45. Albamarla. Le comté d'Aumale (Seine-Maritime, arr. Dieppe, ch.-l. cant.) était alors tenu par Baudouin de Béthune, seigneur de Choques, au nom de son épouse Havoise qui le tenait de son père Guillaume I ${ }^{\mathrm{er}}$.

46. Arches. Arques-la-Bataille, Seine-Maritime, arr. Dieppe, cant. Offranville.

47. Driencort. Driencourt. Nom primitif de Neufchâtel-en-Bray (Seine-Maritime, arr. Dieppe, ch.-1. cant.), pour lequel on trouve aussi Drincourt, Lincourt, voire même Nicourt. Après la construction d'un nouveau château (Novum castrum ou Novum castellum) par Henri Ir d'Angleterre en 1106, la place prend le nom qu'elle porte encore actuellement, même si le nom de Driencourt subsiste concuremment jusqu'au XVI ${ }^{\mathrm{e}}$ siècle. Bibliogr.: BUNEL, Joseph, continué par TOUGARD, Albert, Géographie du département de la Seine Inférieure, p. 78.

48. Belveeir. Beauvoir-en-Lyons, Seine-Maritime, arr. Dieppe, cant. Argueil.

49. Du Moulin, Gabriel, Histoire générale de Normandie, p. 481, § XII, indique par erreur: "depuis la S. Michel." 
Gilles agira de même. Et si le comte de Saint-Gilles veut participer à cette paix, nous ne ferons ni tort ni guerre audit comte, pour autant que ledit comte veuille bien agir envers le roi de France.

Le comte de Périgord ${ }^{50}$ aura la terre qu'il possédait lorsqu'il nous a abandonné; et de même, le vicomte de Brosse ${ }^{51}$ et le comte d'Angoulême ${ }^{52}$ récupèreront leurs terres. Leurs hommes et eux trois nous rendront hommage et service comme devant.

Le vicomte de Turenne tiendra du roi de France ce qu'il doit, et de nous ce qu'il doit.

Le sort de Fortanié de Gourdon ${ }^{53}$ sera le suivant: si nous pouvons prouver par le serment de vingts ou trente hommes légitimes que nous avons tenu les deux châteaux de Cazals ${ }^{54}$ et Peyrilles ${ }^{55}$ pendant une année et un jour, et davantage, et que nous les avons donné audit Fortuné, et si nous voulons les avoir, notre seigneur le roi de France ne pourra s'y opposer.

50. Comes Petragore. Hélie V dit Talleyrand, comte de Périgord, fils et successeur de Boson III († 1166). Par son mariage en 1167 avec Mahaut de Turenne, il devint le beau-frère de Raymond III (cf. note 33). Opposant à Richard Coeur-de-Lion, il fut toujours le champion du roi de France auquel il porta son hommage en 1204. Il mourut en Terre sainte en 1205. Bibliogr.: L'Art de vérifier les dates... par un religieux bénédictin de la congrégation de St-Maur, t. II, Paris, 1787, p. 377-378; Europäische Stammtafeln, Band III (4), Tafel766.

51. Vicecomes Brocie. Bernard III de Brosse, vicomte de Brosse, beau-frère, par son mariage avec Almodis, d'Adhémar, comte d'Angoulême (cf. infra note 45); il fit serment d'allégeance à PhilippeAuguste en 1194. Bibliogr.: BRIAL, Michel-Jean-Joseph (Dom), Recueil des historiens des Gaules et de la France, XVII, p. 44, note i; Europäische Stammtafeln, Band III (4), Tafel 818.

52. Comes Engolismensis. Adhémar, comte d'Angoulême ( $† 16$ juin 1202), fils de Guillaume VI Taillefer (1140-1179) et de Marguerite de Turenne (†1173), fille de Raymond I ${ }^{\text {er }}$ de Turenne et par là grandtante de Raymond III (cf. note 33) et de Mahaut, comtesse de Périgord (cf. note 43). Il épousa en 1186 Alix de Courtenay $(\dagger 1245)$, petite-fille, par son père, Pierre $I^{\text {er }}$ de Courtenay $(\dagger 1183)$, de Louis VI le Gros, roi de France (1108-1137), et qui lui donna Isabelle († 1246), femme de Jean-sans-Terre. Bibliogr. : Europäische Stammtafeln, Band III (4), Tafel 818.

53. Fortunatus de Gorduno. Fortanié de Gourdon (Lot, ch.-l. arr.), issu d'une antique maison remontant à Aymeric, à qui Raymond $\mathrm{I}^{\mathrm{er}}$, comte de Rouergue fit don de Gourdon par son testament en date du 6 septembre 961, fils de Guillaume de Gourdon, devint seigneur de Gourdon en 1195. En épousant en 1179 ou 1180, Aquilina, fille d'Aymar V, vicomte de Limoges (1148-1199) et petitefille par sa mère, Sarah de Cornouailles $(\dagger 1216)$ d'Henri I ${ }^{\text {er }}$ Beauclerc, il devint cousin germain d'Henri II Plantagenêt. Il mourut en 1197 en défendant Cazals (cf. infra note 47) et Peyrilles (cf. infra note 48), assiégés par Richard Coeur-de-Lion. Son fils Bertrand lui succéda et tua, dit-on, Richard à Châlus (6 avril 1199). Bibliogr.: Combarieu, Louis, et CANGARDEL, François, "Gourdon et ses seigneurs du X $\mathrm{X}^{\mathrm{e}}$ au XIV ${ }^{\mathrm{e}}$ s. », Bulletin de la Société des études du Lot, t. 6 (1880), p. 141 et sq.; Combarieu, Louis, Dictionnaire des communes du Lot, Cahors, A. Laytou, 1881, p. 104. DU Moulin, Gabriel, Histoire générale de Normandie, p. 481, § XIV, traduit par: "Fortunat de Gorde".

54. Caseaus. Cazals, Lot, arr. et cant. Cahors.

55. Perillac. Peyrilles, Lot, arr. Gourdon, cant. Saint-Germain-du-Belair. 
Pour la construction de la demeure du château neuf de Tours ${ }^{56}$, nous nous en remettrons au conseil de l'archevêque de Reims ${ }^{57}$ et au conseil de Dreu de Mello ${ }^{58}$.

Pour les Andelys ${ }^{59}$, il en sera ainsi : ni Monseigneur le roi de France, ni nousmêmes n'en réclamerons le fief ou la propriété. Et s'il arrive que l'archevêque de Rouen envoie une sentence d'interdit ou d'excommunication sur la terre du roi de France ou des siens, le seigneur roi de France pourra confisquer les Andelys, ce que l'archevêque y possède et ses dépendances, jusqu'à ce que deux diacres, ou prêtres, que le roi de France aura choisi en bonne foi, sous serment, et deux diacres, ou prêtres, que nous aurons choisis de même, décident si l'interdit ou l'excommunication ont été jetés justement ou injustement. S’ils décident que cela fut juste, le roi de France rendra les Andelys audit archevêque et ce qu'il aura perçu pendant ce temps, et il expiera selon le jugement des arbitres. Mais s'ils décident que cela fut jeté injustement, tout ce que le roi de France aura levé sur les Andelys et ses dépendances sera perdu pour l'archevêque, et l'archevêque lèvera l'interdit ou l'excommunication. Il en sera de même pour nous. Si l'un desdits arbitres décède, d'un côté ou de l'autre, un autre sera nommé à la place du défunt, au terme du même serment. Si l'archevêque meurt, les revenus des Andelys et de ses dépendances iront au chapitre de Notre-Dame de Rouen, jusqu'à ce qu'un autre archevêque lui succède. Et nous, nous ne causerons aucun tort auxdits arbitres à cause de leur sentence. Les Andelys ne pourront être fortifiés.

Quant à nous, nous tiendrons pour quitte notre seigneur le roi de France de tous les biens qu'il a pris aux églises de notre terre, et qui sont sur la sienne. Le roi de France fera de même pour nous. Désormais, ni nous, ni le roi de France, si une guerre éclatait, ne prendrions ni reprendrions des biens des églises de la terre de l'autre. Et les églises jouiront de part et d'autre des mêmes paix et liberté qu'avant la guerre.

Ensuite, nous ne nous opposerons pas aux hommes du roi de France, ni aux fiefs qui dépendent de lui. Il agira de même envers nous, sauf les services que nous devons audit roi de France, pour les fiefs que nous tenons de lui, comme les vassaux doivent les rendre. Nous ne recevrons de plus aucun homme lige du roi de France contre lui, tant qu'il vivra, ni lui nos homme liges, contre nous, tant que nous vivrons.

Après l'accomplissement de ces clauses, le comte de Leicester, tous les prisonniers et tous les otages des prisonniers, comme cela a été décidé, seront libérés de part et

56. Castelli novi Turonis. Tours, Indre-et-Loire, ch.-1.

57. Guillaume I ${ }^{\text {er }}$ de Champagne, dit «aux Blanches mains », archevêque de Reims (1176-1205).

58. Drogo de Melloto. Dreu de Mello (1130-1218), connétable de France en 1193.

59. Andeliacum. Les Andelys, Eure, ch.-1. arr. 
d'autre. Nous jurons de bonne foi que nous observerons cela et le roi de France de même.

Pour que toutes les choses susdites demeurent fermes et stables, nous les confirmons par notre sceau. Fait entre Gaillon et le Vaudreuil, l'an du Verbe incarné mil cent quatre-vingt-quinze. 